\title{
Diagnosis and Treatment of Human Salmonellosis in Addis Ababa City, Ethiopia
}

\author{
Legesse Garedew ${ }^{1 D},{ }^{1}$ Semaria Solomon, ${ }^{1}$ Yoseph Worku, ${ }^{1}$ Hilina Worku, ${ }^{1}$ \\ Debela Gemeda, ${ }^{1}$ Gada Lelissa, ${ }^{1}$ Yeshwondm Mamuye, ${ }^{1}$ Rajiha Abubeker, ${ }^{2}$ \\ Amete Mihret ${ }^{(D},{ }^{2}$ Surafel Fentaw, ${ }^{2}$ Abeibe Worku, ${ }^{3}$ Melish Bahiru, ${ }^{4}$ and Girume Erenso ${ }^{1}$ \\ ${ }^{1}$ St Paul's Hospital Millennium Medical College, Addis Ababa, Ethiopia \\ ${ }^{2}$ Ethiopian Public Health Institute, Addis Ababa, Ethiopia \\ ${ }^{3}$ Alem Tena Clinic, Addis Ababa, Ethiopia \\ ${ }^{4}$ Selam Clinic, Addis Ababa, Ethiopia \\ Correspondence should be addressed to Legesse Garedew; legesse_lg@yahoo.com
}

Received 18 November 2017; Accepted 19 April 2018; Published 23 May 2018

Academic Editor: Ernesto S. Nakayasu

Copyright (C) 2018 Legesse Garedew et al. This is an open access article distributed under the Creative Commons Attribution License, which permits unrestricted use, distribution, and reproduction in any medium, provided the original work is properly cited.

Background. Diagnosis using reliable tools and treatment following in vitro antimicrobial susceptibility tests are critical to proper addressing of antibiotic-resistant Salmonella infection. Methodology. A cross-sectional study was conducted to assess the practice of diagnosis and treatment of salmonellosis in Addis Ababa. Tube Widal test (for blood samples only), culture, biochemical and carbohydrate fermentation, serotyping, and antimicrobial susceptibility tests were employed for both blood and stool samples. Results. Of all the diseases listed in the diagnosis, nontyphoidal $(n=72,13.71 \%)$ and typhoidal $(n=47,8.95 \%)$ salmonellosis were the second and third common diseases. Among the 288 blood samples, almost half were positive for $\mathrm{O}, \mathrm{H}$, or both antigens. However, only $1(0.68 \%)$ of the positive blood samples yielded Salmonella isolate during culture. The study demonstrated low specificity $(0.68 \%)$ and positive predictive value (48.78\%) of Widal test. Conversely, the test showed $100 \%$ sensitivity and negative predictive values. Salmonella isolates were identified from 7 (7.07\%) of 99 stool samples. Two-thirds of salmonellosis suspected patients received antibiotic treatment. However, only half of the confirmed salmonellosis patients were treated with appropriate antibiotics. All of the isolates were susceptible to ciprofloxacin and ceftriaxone but resistant to ampicillin. Conclusions. Majority of the patients who participated in this study were wrongly diagnosed using symptoms, clinical signs, and tube Widal test. Consequently, most of the patients received inappropriate treatment.

\section{Introduction}

Salmonellosis continues to be among the major causes of illnesses and deaths around the globe particularly in developing countries [1-3]. Salmonella enterica subspecies I bacteria are the causes of the disease and over 93 million new infections worldwide each year [4]. World Health Organization (WHO) estimated 3 million Salmonella-associated deaths globally per year [5]. Diseases caused by Salmonella in humans are gastroenteritis or nontyphoidal salmonellosis (by Salmonella Typhimurium or Salmonella Enteritidis) [4] and typhoid fever (by Salmonella Typhi or Paratyphi) [6]. Global nontyphoidal salmonellosis burden estimate indicated 155,000 deaths each year [4]. In Sub-Saharan Africa, the invasive nontyphoidal salmonellosis often causes life-threatening bacteremia for about 1.9 million immunosuppressed individuals $[7,8]$.

Despite the high global burden of salmonellosis, wellestablished diagnosis and treatment have only recently begun to advance [7]. Since the clinical pictures of the disease are nonspecific; confirmed diagnosis relies on the gold standard test (culture) [9]. But culture is expensive, labor-intensive, and time-consuming [10]. In many typhoid fever endemic areas where culture facilities are not available, Widal test is considered to be the only quick diagnostic tool [11]. Though the Widal test is no longer being used as a diagnostic tool in developed nations due to its low sensitivity and specificity, 
it is still commonly in use in developing countries such as Ethiopia [9, 11]. Widal test is the rapid and affordable diagnostic assay available in Africa and it has been extensively employed since typhoid fever is endemic in most of the continent [12]. In the case of nontyphoidal salmonellosis, clinical signs and symptoms are still the main means of diagnosis [13].

Reports have shown increased typhoidal salmonellosis cases resistant to many drugs such as fluoroquinolones. In countries with a higher incidence of multidrug-resistant typhoidal Salmonella isolates, susceptibility to ciprofloxacin and other fluoroquinolones is reported between $44 \%$ and 59\% [14]. Based on the National Antimicrobial Resistance Monitoring System survey from 2005 to 2006, 84\% of nontyphoid Salmonella clinical isolates displayed multidrug resistant phenotype and $4.1 \%$ of the isolates showed reduced susceptibility to cephalosporins in the United States of America [15]. A surveillance study in Europe from 2000 to 2004 on nontyphoid Salmonella also showed that $15 \%$ of the isolates displayed multidrug resistant phenotype and $20 \%$ of the isolates showed resistance to nalidixic acid [16].

In Ethiopia, the diagnosis of typhoidal and nontyphoidal salmonellosis has been carried out using the Widal test and clinical pictures, respectively [17]. But the importance of both diagnostic methods has been strongly criticized [11]. Hence, evaluation of the effectiveness of these methods in the diagnosis of salmonellosis against the gold standard method is necessary for better patient outcomes. The main objective of this study was to examine the level of agreement between results of Widal test and clinical examinations with the results of culture method in the diagnosis of salmonellosis. In addition, the study aimed at assessing the appropriateness of treatment for the diagnosis and antimicrobial susceptibility pattern of Salmonella isolates.

\section{Materials and Methods}

2.1. Study Area, Sample Size, and Sampling Procedure. A cross-sectional study was carried out on salmonellosis suspected patients who visited Selam (public) and Alem Tena (private) clinics in Addis Ababa, Ethiopia, from August 2015 to March 2016. The sample size was determined using a single population proportion formula, $n=p(1-p)(Z \alpha / 2) 2 / d 2$ [18] with the following assumptions: $Z \alpha / 2=1.96$ for the standard scale of $95 \%$ level of confidence, level of precision $(d)=$ $5 \%$, and $p=0.5$ since there was no previous study carried out on this issue in the current study health facilities. Ten percent nonresponse rate was considered. Study subjects who visited the two clinics and fulfilled the eligibility criteria were enrolled after receiving their written consent. Though the calculated sample population was 423 , only 387 eligible and willing patients participated in the current study. Case history study was conducted on 367 patients since the remaining 20 patients' record could not be found in the study clinics. Sociodemographic data was gathered using structured and pretested questionnaire and patients' clinical records. All the febrile $(n=288)$ and diarrheic $(n=99)$ patients provided blood and stool samples, respectively. Stool specimens were collected using labeled wide-mouthed plastic containers and clean wooden applicator sticks. Venous blood samples, 8-10 $\mathrm{ml}$ from adults and 3-5 $\mathrm{ml}$ from children, were collected aseptically. All of the samples were collected during the first day of patients' examination in the study clinics.

\subsection{Sample Analysis}

2.2.1. Blood Samples Analysis Using Widal Test. The Widal tube agglutination test (titration) was performed as described previously [9] using serial dilution of serum samples in $0.95 \%$ fresh saline preparation from $1: 20$ to $1: 640$ for anti-TO and anti-TH separately. Subsequently, a drop of $\mathrm{O}$ and $\mathrm{H}$ antigens was added to all test tubes. Antibody titer $>1: 80$ for antiTO and >1:160 for anti-TH were taken as a cut-off value to indicate a recent infection with typhoid fever as per the manufacturer's manual (Chromatest Febrile Antigens kits, Linear Chemicals, Spain).

2.2.2. Bacteriological Investigation of Blood Sample. All blood samples $(n=288)$ were collected before administration of any drug and after checking that patients did not take any form of antibiotics at least for the last 2 weeks. The bacteriological examination was carried out based on standard procedures $[19,20]$ with minor modifications. Briefly, blood samples were immediately inoculated into tryptic soy broth (Abtek USP, Abtek Biologicals Ltd., England) and transported to the Ethiopian Public Health Institute (EPHI) laboratory within 2-4 hours of collection. Upon arrival, culture bottles were incubated aerobically at $37^{\circ} \mathrm{C}$ for $5-7$ days. Examination of incubated broths for growth indicators was conducted on daily basis and those showing growth were subcultured onto MacConkey (Oxoid, England) and blood (Oxoid, England) agar plates. The plates were incubated at $37^{\circ} \mathrm{C}$ in $5 \% \mathrm{CO}_{2}$ incubator for a minimum of 48 hours and examined daily for the presence of distinct colonies. Bacterial identification was carried out based on colony morphology and Gram staining characteristics. Identified Salmonella isolates were further confirmed using biochemical, carbohydrates fermentation, and serotyping analysis.

2.2.3. Stool Sample Analysis. Stool samples $(n=99)$ were submitted to the EPHI laboratory in sterile and leak-proof containers within 2 hours of collection and processed immediately according to the established protocol [21]. Approximately 1.5-2 grams of stool, preferably with blood and mucus, was mixed with Selenite F (TM Media, India) enrichment broth media and incubated for 24 hours at $37^{\circ} \mathrm{C}$. Subculturing was carried out on MacConkey and Salmonella-Shigella (SS) agars (Oxoid, England) at $37^{\circ} \mathrm{C}$ for 24 hours. Non-lactose fermenter colonies on MacConkey and SS agars with black center on SS agar were subjected to biochemical, carbohydrates fermentation, and serotyping tests.

2.2.4. Biochemical, Carbohydrate Fermentation, and Serotyping Tests. Presumptive Salmonella colonies were subjected to biochemical and carbohydrates fermentation tests including triple sugar iron agar (TSIA) (TM Media, India), lysine iron agar (LIA) (Oxoid, England), indole (TM Media, India), citrate (TM Media, India), and urease (Oxoid, England) tests 
as described earlier $[19,20,22]$. Poly antisera Salmonella O, antiserum poly A-I, and Vi (Difco ${ }^{\mathrm{TM}}$ Salmonella O, BD, Ireland) were used during serotyping for confirmation. Colonies showed an alkaline slant with acid butt and hydrogen sulfide production on TSIA, positive for lysine on LIA, positive for citrate utilization on Simmons' citrate agar, negative for urea hydrolysis on urea agar, and negative for indole test on SIM medium and that agglutinate on tube agglutination test with Salmonella polyvalent antisera was considered as Salmonella isolate.

\subsubsection{Antimicrobial Susceptibility Test. All isolates were sub-} jected to antimicrobial susceptibility test using Kirby-Bauer disk diffusion method. The bacterial suspension was prepared by taking $4-5$ pure isolated colonies into trypticase soy yeast broth (Abtek Biologicals Ltd., England) and adjusted to 0.5 McFarland turbidity standards [23]. The suspension was transferred to Mueller-Hinton agar plate using a sterile cotton swab. Plates were uniformly inoculated by rubbing the swab against the entire agar surface and rotating plates at about 60 degrees 3 times. Antibiotic-impregnated disks were applied to the surface of the inoculated plates using sterile forceps and incubated aerobically at $37^{\circ} \mathrm{C}$ for 24 hours. Antibiotics were selected based on common prescription trend and availability of antibiotic disks. Salmonella isolates were tested for antibiotics (Oxoid, England) including ampicillin (AMP, $10 \mu \mathrm{g}$ ), ceftriaxone (CRO, $30 \mu \mathrm{g})$, chloramphenicol (C, $30 \mu \mathrm{g})$, and ciprofloxacin (CIP, $5 \mu \mathrm{g})$.

2.2.6. Quality Control and Data Analysis. Sterility and performance tests were performed for all media prior to the tests. S. Typhimurium (ATCC 13311), Escherichia coli (ATCC 25922), and Staphylococcus aureus (ATCC 25923) were used as quality control species. Data were double entered, cleaned, and analyzed using Microsoft Excel Spreadsheet version 2016 16.0.6741.2048.

2.2.7. Ethical Considerations. Ethical clearance was obtained from Institutional Board of Review of St. Paul's Hospital Millennium Medical College (protocol no. P. M23/72, on 17 February 2015). Written informed consent was obtained from all study participants. Participants also gave their written agreement that the research result will be published with keeping their identity anonymous. Salmonellosis suspected patients with antimicrobial treatment history within two weeks were excluded from the study. Information obtained during the course of the study was kept confidential and communicated to the clinics.

\section{Results}

3.1. Sociodemographic Characteristics of the Study Participants. Clinical records were found for 367 of salmonellosis suspected patients. The remaining 20 patients were registered as referral cases from other health facilities for Widal test purpose only. Among patients with complete records, 5.44\% $(n=20)$ and $2.72 \%(n=10)$ were under 10 and over 70 years, respectively. About $37 \%(n=135)$ of the patients were in
21-30 years of age group. Majority of the patients were from economically productive age group (18-49 years). Close to $60 \%(n=220)$ of the participants were males.

3.2. Diagnosis of Salmonellosis. According to the reviewed clinical records of the study clinics, the diagnosis of salmonellosis is carried out mainly based on clinical signs and symptoms. Of the total diseases listed as primary and secondary diseases, acute febrile illness was the most frequently mentioned disease $(n=208,39.62 \%)$ as shown in Table 1 . Nontyphoidal salmonellosis was the second common disease $(n=72,13.71 \%)$ of all diseases documented. Typhoid fever is reported as the second $(n=43,11.72 \%)$ and the least $(n=4$, $1.09 \%)$ common disease under the primary and secondary diseases categories, respectively. In general, typhoid fever is among the top three common diseases recorded in the current study. Infectious diseases showing similar clinical signs and symptoms with typhoid fever such as acute febrile illness and unspecified infectious diseases were also the frequently listed diseases.

3.3. Widal Test, Culture, and Serotyping. Of 288 blood samples examined using tube Widal test, 51.39\% $(n=148)$ were positive for $\mathrm{O}, \mathrm{H}$, or both antigens as tabulated in Table 2 . Antibody titers of $\geq 1: 80$ for anti-TO and $\geq 1: 160$ for anti $\mathrm{TH}$ were taken as a cut-off value to indicate recent infection of typhoid fever. In the current study, tube Widal test demonstrated $100 \%$ sensitivity as well as negative predictive values for both $\mathrm{O}$ and $\mathrm{H}$ antigens. Conversely, it indicated very poor specificity $(0.68 \%)$ and positive predictive values $(48.78 \%)$. There was a good level of agreement $($ Kappa $=0.62)$ in detecting both antigens. Out of 148 blood samples positive for O, H, or both antigens, only 1 sample yielded Salmonella isolate. No Salmonella isolate was identified from Widal test negative blood samples. Among the 99 stool sample cultures, 7 yielded Salmonella isolates. Of 387 cultured blood and stool samples, only 8 produced Salmonella isolates. Six of the isolates were identified from diarrheic/dysentery patients and the remaining 2 were from acute febrile illness and unspecified infectious disease patients. All Salmonella isolates were confirmed using serotyping.

3.4. Antibiotic Treatment and Susceptibility Patterns. Nearly $67 \%(n=245)$ of salmonellosis suspected patients were treated with one or more antibiotics. Among patients who received antibiotics treatment, $49.8(n=122), 25.3(n=62)$, $9.8(n=24)$, and $7.76 \%(n=19)$ took ciprofloxacin, doxycycline, metronidazole, and cotrimoxazole, respectively, as shown in Table 3. All the isolates were susceptible to ciprofloxacin and ceftriaxone but resistant to ampicillin.

\section{Discussion}

Majority of the participants of the current study were from economically productive age group (18-49 years). This finding is in disagreement with studies that reported that less than 5 years of age is the highly affected segment of the population $[24,25]$. Putting the diagnosis in a broad category like acute 
TABLE 1: List of diseases and treatment trend (with antibiotics and/or anthelmintics) in selected clinics, Addis Ababa, Ethiopia.

\begin{tabular}{|c|c|c|c|c|c|c|c|}
\hline \multirow{2}{*}{ Name of the diseases } & \multirow{2}{*}{ Category } & \multicolumn{2}{|c|}{ As primary disease } & \multicolumn{2}{|c|}{ As secondary disease } & \multicolumn{2}{|c|}{ Total } \\
\hline & & Freq. & $\%$ & Freq. & $\%$ & Freq. & $\%$ \\
\hline \multirow{2}{*}{ Acute febrile illness } & Total & 185 & 50.40 & 23 & 6.27 & 208 & 39.62 \\
\hline & Treated & 129 & 69.73 & 19 & 82.61 & 148 & 71.15 \\
\hline \multirow{2}{*}{ Diarrhea/Dysentery } & Total & 53 & 14.44 & 19 & 5.18 & 72 & 13.71 \\
\hline & Treated & 45 & 84.91 & 3 & 15.79 & 48 & 66.67 \\
\hline \multirow{2}{*}{ Typhoid fever } & Total & 4 & 1.09 & 43 & 11.72 & 47 & 8.95 \\
\hline & Treated & 4 & 100 & 40 & 93.02 & 44 & 93.62 \\
\hline \multirow{2}{*}{ Unspecified infectious diseases } & Total & 39 & 10.63 & 6 & 1.63 & 45 & 8.57 \\
\hline & Treated & 29 & 74.36 & 1 & 16.67 & 30 & 66.67 \\
\hline \multirow{2}{*}{ Dyspepsia } & Total & 17 & 4.63 & 12 & 3.26 & 29 & 5.52 \\
\hline & Treated & 15 & 88.24 & 9 & 75.00 & 24 & 82.76 \\
\hline \multirow{2}{*}{ Intestinal parasitism } & Total & 5 & 1.36 & 22 & 5.99 & 27 & 5.14 \\
\hline & Treated & 5 & 100 & 19 & 86.36 & 24 & 88.89 \\
\hline \multirow{2}{*}{ Urinary tract infection } & Total & 10 & 2.72 & 15 & 4.09 & 25 & 4.76 \\
\hline & Treated & 10 & 100 & 14 & 93.33 & 24 & 96.00 \\
\hline \multirow{2}{*}{ Respiratory tract diseases } & Total & 12 & 3.27 & 10 & 2.72 & 22 & 4.19 \\
\hline & Treated & 11 & 91.67 & 9 & 90.00 & 20 & 90.91 \\
\hline \multirow{2}{*}{ Others } & Total & 23 & 6.27 & 27 & 7.37 & 50 & 9.52 \\
\hline & Treated & 18 & 78.26 & 25 & 92.59 & 43 & 86 \\
\hline \multirow{2}{*}{ No diagnosis recorded } & Total & 19 & 5.18 & 190 & 51.77 & 18 & 100 \\
\hline & Treated & 2 & 10.53 & 32 & 16.84 & 1 & 5.56 \\
\hline \multirow{3}{*}{ Total } & Patients Recorded & 367 & 100 & 367 & 100 & 367 & 100 \\
\hline & Diagnosis recorded & 348 & 94.82 & 177 & 48.23 & 525 & 100 \\
\hline & Treated & 268 & 73.02 & 171 & 46.59 & 439 & 83.62 \\
\hline
\end{tabular}

TABLe 2: Widal test result $(n=288)$ in selected clinics, Addis Ababa, Ethiopia.

\begin{tabular}{|c|c|c|c|}
\hline Test & Result & Freq. & $\%$ \\
\hline \multirow{2}{*}{ Widal test against Salmonella $\mathrm{H}$ antigen } & $-\mathrm{Ve}$ & 165 & 57.29 \\
\hline & $+\mathrm{Ve}$ & 123 & 42.71 \\
\hline \multirow{2}{*}{ Widal test against Salmonella $\mathrm{O}$ antigen } & $-\mathrm{Ve}$ & 168 & 58.33 \\
\hline & $+\mathrm{Ve}$ & 120 & 41.67 \\
\hline \multirow{5}{*}{ Widal test against both $\mathrm{H}$ and $\mathrm{O}$ antigens of Salmonella } & Both -Ve & 140 & 48.61 \\
\hline & Both $+\mathrm{Ve}$ & 95 & 32.99 \\
\hline & +ve for either $\mathrm{O}$ or $\mathrm{H}$ & 148 & 51.39 \\
\hline & $\mathrm{H}+\mathrm{Ve}$ but $\mathrm{O}-\mathrm{Ve}$ & 25 & 8.68 \\
\hline & $\mathrm{O}+\mathrm{Ve}$ but $\mathrm{H}-\mathrm{Ve}$ & 28 & 9.72 \\
\hline
\end{tabular}

febrile illness, diarrhea/dysentery, and unspecified infectious diseases rather than naming specific disease is an indication of lack of clear picture of the disease among physicians.

Culture is the gold standard diagnostic method for Salmonella infection. But it is often unavailable or less commonly applied in low- and middle-income countries like Ethiopia due to time and cost constraints $[1,26]$. Instead, Widal test is still widely used for typhoid fever suspected patients diagnosis despite the fact that it has poor predictive outcome [27]. Clinical signs and symptoms are still the routine diagnostic methods for nontyphoidal salmonellosis in developing countries [28]. Nonspecific names of diseases such as acute febrile illness, diarrhea/dysentery, and unspecified infectious diseases are frequently listed by physicians which might be due to the lack of confirmatory diagnostic tools.

Almost half of the blood samples were positive either for $\mathrm{O}, \mathrm{H}$, or both Salmonella antigens during the Widal test. This result is similar to a report from India [29], lower than from Ethiopia [30], but higher than from Kenya [31]. Culture positive results of samples collected from typhoidal salmonellosis suspected patients in the current and other studies [29-31] are very low compared with Widal test results. This might suggest that there has been overdiagnosis of 
TABLE 3: Antimicrobials prescribed for salmonellosis suspected patients in selected clinics, Addis Ababa, Ethiopia.

\begin{tabular}{lcc}
\hline Treatment & Frequency & Percentage \\
\hline Patients who received & 245 & 66.76 \\
antimicrobial treatment & 122 & $49.8^{*}$ \\
Ciprofloxacin & 62 & $25.3^{*}$ \\
Doxycycline & 24 & $9.8^{*}$ \\
Metronidazole & 19 & $7.76^{*}$ \\
Cotrimoxazole & 5 & $2.04^{*}$ \\
Amoxicillin & 4 & $1.63^{*}$ \\
Ceftriaxone & 9 & $3.67^{*}$ \\
Other antibiotics & 122 & 33.24 \\
Patients who received no & & 100 \\
antimicrobial treatment & 367 & \\
\hline
\end{tabular}

${ }^{*}$ Percentage of patients who received antimicrobial treatment.

typhoid fever due to the very poor specificity of Widal test $[9,11,27,29]$.

In this study, the identification of Salmonella from both blood and stool samples is lower than reports from Ethiopia $[3,17,30]$. The variation might be partly attributed to difference in study settings since the current study is conducted at clinics but the previous studies were carried out at hospitals where prolonged febrile illness patients are found. The very poor positive predictive value and low specificity of Widal test results of the current research are in agreement with previous studies in Ethiopia [17, 30] and other countries [29, 32]. Since positive predictive value is considered as one of the most important clinical diagnostic tools $[17,30]$, very low positive predictive value in the current research indicates that Widal test was not reliable for the diagnosis of the disease in the study settings. The very high false positive result of the Widal test in this research is an indication that patients are wrongly diagnosed and treated as typhoid fever patients. This inappropriate treatment based on tube Widal test results and clinical signs and symptoms might have created unnecessary treatment costs, pressure on the normal flora of patients, and favorable condition for antimicrobial resistance strains development $[4,30]$. Most importantly, highly fatal diseases of febrile nature might be missed out and ultimately lead to poor prognosis.

The current prevalence of nontyphoidal salmonellosis as confirmed by culture and serotyping is comparable with previous studies in Ethiopia [33] and in Sub-Saharan Africa [34]. The relatively higher prevalence of nontyphoidal salmonellosis compared with typhoidal salmonellosis in this study is in agreement with the report that suggested the proportion of typhoidal Salmonella infection tends to decline since 1970s while nontyphoidal Salmonella infection shows a relative increase in Ethiopia [33]. The current nontyphoidal Salmonella infection prevalence might be lower than its actual occurrence since many patients in developing countries do not seek medical attention for less severe gastroenteritis. They are unable to cover medical costs even for severe cases. Widespread malnutrition, higher HIV-AIDS prevalence, close relationship with animals, the common practice of backyard animal slaughtering, unhygienic food and water handling, raw meat consumption habits, and population crowd are suggestive of its higher prevalence than reported in the current study.

Nowadays, drug resistant Salmonella infection is one of the critical problems of the globe [35]. As demonstrated by research results $[2,36-38]$ and summarized by the recent review [39], there is an increasing prevalence of drug resistant Salmonella isolates in Ethiopia. The resistance of all Salmonella isolates against ampicillin in the current study, which is higher than reports in Ethiopia [2, 36, 38, 39], is an indication of worsening situation of antimicrobial resistance Salmonella in the country.

Due to the increase in the resistance against ampicillin, ciprofloxacin continues to be widely used globally in the treatment of salmonellosis [1]. Matching with the global trend, this study revealed that ciprofloxacin is the most frequently prescribed drug followed by doxycycline and metronidazole in the study clinics. This treatment regimen seems appropriate since all Salmonella isolates were susceptible to ciprofloxacin. But only half of the confirmed salmonellosis patients were treated with appropriate antibiotics. Vigilant use of ciprofloxacin is needed since resistant isolates are reported from countries in Europe, Asia, Africa, and the United States [8].

\section{Conclusions}

The general prevalence of salmonellosis reported in the current study is low. This low culture proven salmonellosis prevalence demonstrated that the vast majority of patients were wrongly diagnosed using clinical signs and symptoms and Widal test. The wrong diagnosis was also followed by incorrect treatment that might have had a grave consequence on the patients' prognosis. This situation might have also posed a high risk for antimicrobial resistance strains development. Since the Widal test is found to be inappropriate as a diagnostic tool for typhoidal salmonellosis in endemic areas like Ethiopia, it should be either replaced with more accurate tests or followed by alternative diagnostic tools. The resistance of all Salmonella isolates against ampicillin is an indication that ampicillin is no more effective in the treatment of salmonellosis. Vigilant prescription of ciprofloxacin for salmonellosis patients is encouraged. The nationwide indepth investigation regarding the prevalence of the disease, circulating serotypes, their antimicrobial susceptibility patterns, and appropriate diagnostic options is strongly recommended.

\section{Disclosure}

This research did not receive any specific grant from funding agencies in the public, commercial, or not-for-profit sectors.

\section{Conflicts of Interest}

The authors declare that they have no conflicts of interest. 


\section{Authors' Contributions}

Legesse Garedew carried out the conception of the research idea and design of the methodology. In addition, Legesse Garedew performed laboratory work, data collection, result analysis and interpretation, and preparation of the manuscript for publication. Girume Erenso was involved in enriching the idea, designing methods, and data collection and analysis. Semaria Solomon and Rajiha Abubeker contributed to the shaping of the research idea and methodology design. Rajiha Abubeker, Amete Mihret, Surafel Fentaw, Abeibe Worku, and Melish Bahiru carried out the laboratory analysis. Yeshwondm Mamuye, Hilina Worku, Debela Gemeda, Gada Lelissa, and Yeshwondm Mamuye reviewed the proposal and the manuscript. All researchers participated in manuscript preparation and approved the final manuscript.

\section{Acknowledgments}

St Paul's Hospital Millennium Medical College is greatly acknowledged for all rounded support towards the smooth accomplishment of this research. The authors are also grateful for the support of the of Ethiopian Public Health Institute and Selam and Alem Tena Clinics. The authors are deeply grateful to participants of the research.

\section{References}

[1] J. A. Crump and E. D. Mintz, "Global trends in typhoid and paratyphoid fever," Clinical Infectious Diseases, vol. 50, no. 2, pp. 241-246, 2010.

[2] L. Garedew-Kifelew, N. Wondafrash, and A. Feleke, "Identification of drug-resistant Salmonella from food handlers at the University of Gondar, Ethiopia," BMC Research Notes, vol. 7, article no. 545, 2014.

[3] G. Beyene, S. Nair, D. Asrat, Y. Mengistu, H. Engers, and J. Wain, "Multidrug resistant Salmonella Concord is a major cause of salmonellosis in children in Ethiopia," The Journal of Infection in Developing Countries, vol. 5, no. 1, pp. 23-33, 2011.

[4] S. E. Majowicz, J. Musto, E. Scallan et al., "The global burden of nontyphoidal Salmonella gastroenteritis," Clinical Infectious Diseases, vol. 50, no. 6, pp. 882-889, 2010.

[5] WHO, "Food Safety-Food borne diseases and value chain management for food safety," in Proceedings of the Forging Links between Agriculture and Health, CGIAR on Agriculture and Health Meeting, 2007.

[6] R. Lozano, M. Naghavi, and K. Foreman, "Global and regional mortality from 235 causes of death for 20 age groups in 1990 and 2010: a systematic analysis for the Global Burden of Disease Study 2010," The Lancet, vol. 380, no. 9859, pp. 2095-2128, 1990.

[7] J. S. Gunn, J. M. Marshall, S. Baker, S. Dongol, R. C. Charles, and E. T. Ryan, "Salmonella chronic carriage: Epidemiology, diagnosis, and gallbladder persistence," Trends in Microbiology, vol. 22, no. 11, pp. 648-655, 2014.

[8] S. Kariuki and R. S. Onsare, "Epidemiology and genomics of invasive nontyphoidal Salmonella infections in Kenya," Clinical Infectious Diseases, vol. 61, supplement 4, pp. S317-S324, 2015.

[9] B. Ley, G. Mtove, K. Thriemer et al., "Evaluation of the Widal tube agglutination test for the diagnosis of typhoid fever among children admitted to a rural hdospital in Tanzania and a comparison with previous studies," BMC Infectious Diseases, vol. 10, no. 1, 2010.

[10] S. Hosolgu, V. Bosnak, S. Akalin, M. F. Geyik, and C. Ayaz, "Evaluation of false negativity of the Widal test among culture proven typhoid fever cases," The Journal of Infection in Developing Countries, vol. 2, no. 06, 2008.

[11] L. A. Olopoenia and A. L. King, "Widal agglutination test - 100 years later: Still plagued by controversy," Postgraduate Medical Journal, vol. 76, no. 892, pp. 80-84, 2000.

[12] E. Mweu and M. English, "Typhoid fever in children in Africa," Tropical Medicine \& International Health, vol. 13, no. 4, pp. 532540, 2008.

[13] J. R. Verani, S. Toroitich, J. Auko et al., "Burden of invasive nontyphoidal salmonella disease in a rural and urban site in Kenya, 2009-2014," Clinical Infectious Diseases, vol. 61, pp. S302S309, 2015.

[14] R. L. Ochiai, C. J. Acosta, M. C. Danovaro-Holliday et al., "A study of typhoid fever in five Asian countries: disease burden and implications for controls," Bulletin of the World Health Organization, vol. 86, no. 4, pp. 260-268, 2008.

[15] J. A. Crump, F. M. Medalla, K. W. Joyce et al., "Antimicrobial resistance among invasive nontyphoidal Salmonella enterica isolates in the United States: National Antimicrobial Resistance Monitoring System, 1996 to 2007," Antimicrobial Agents and Chemotherapy, vol. 55, no. 3, pp. 1148-1154, 2011.

[16] S. Meakins, I. S. T. Fisher, C. Berghold et al., "Antimicrobial drug resistance in human nontyphoidal Salmonella isolates in Europe 2000-2004: A report from the Enter-net international surveillance network," Microbial Drug Resistance, vol. 14, no. 1, pp. 31-35, 2008.

[17] G. Andualem, T. Abebe, N. Kebede, S. Gebre-Selassie, A. Mihret, and H. Alemayehu, "A comparative study of Widal test with blood culture in the diagnosis of typhoid fever in febrile patients," BMC Research Notes, vol. 7, no. 1, article no. 653, 2014.

[18] E. R. Ziegel, S. Lemeshow, D. Hosmer, J. Klar, and S. Luanga, "Adequacy of Sample Size in Health Studies," Technometrics, vol. 36, no. 2, p. 232, 1994.

[19] L. S. Garcia, Clinical microbiology procedures handbook, American Society for Microbiology Press, 2010.

[20] E. Baron et al., Cumulative Techniques and Procedures in Clinical Microbiology (Cumitech) 1C: Blood Cultures IV, ASM Press, 2005.

[21] J. Vandepitte, K. Engbaek, P. P. Rohner, H. Peter, and C. Claus, Basic Laboratory Procedures in Clinical Bacteriology, World Health Organization, Geneva, Switzerland, 2nd edition, 2003.

[22] N. M. Moore, "Color Atlas of Medical Bacteriology," Laboratory Medicine, vol. 44, no. 4, pp. el16-e117, 2013.

[23] J. H. Jorgensen and J. D. Turnidge, "Susceptibility test methods: dilution and disk diffusion methods," Manual of Clinical Microbiology, Eleventh Edition, American Society of Microbiology, pp. 1253-1273, 2015.

[24] S. C. Morpeth, H. O. Ramadhani, and J. A. Crump, "Invasive non-typhi Salmonella disease in Africa," Clinical Infectious Diseases, vol. 49, no. 4, pp. 606-611, 2009.

[25] D. T. Leung, S. K. Das, M. A. Malek et al., "Non-typhoidal Salmonella gastroenteritis at a diarrheal hospital in Dhaka, Bangladesh, 1996-2011," The American Journal of Tropical Medicine and Hygiene, vol. 88, no. 4, pp. 661-669, 2013.

[26] J. A. Crump, M. Sjölund-Karlsson, M. A. Gordon, and C. M. Parry, "Epidemiology, clinical presentation, laboratory diagnosis, antimicrobial resistance, and antimicrobial management of 
invasive Salmonella infections," Clinical Microbiology Reviews, vol. 28, no. 4, pp. 901-937, 2015.

[27] A. Adhikari, R. Rauniyar, P. P. Raut, K. D. Manandhar, and B. P. Gupta, "Evaluation of sensitivity and specificity of ELISA against Widal test for typhoid diagnosis in endemic population of Kathmandu," BMC Infectious Diseases, vol. 15, no. 1, article no. 523, 2015.

[28] A. Marzel et al., "Persistent Infections by Nontyphoidal Salmonella in Humans: Epidemiology and Genetics," Clinical Infectious Diseases, vol. 62, no. 7, pp. 879-86, 2016.

[29] D. Narayanappa, R. Sripathi, K. Jagdishkumar, and H. S. Rajani, "Comparative study of dot enzyme immunoassay (Typhidot$\mathrm{M})$ and Widal test in the diagnosis of typhoid fever," Indian Pediatrics, vol. 47, no. 4, pp. 331-333, 2010.

[30] A. G. wasihun, L. N. Wlekidan, S. A. Gebremariam et al., "Diagnosis and Treatment of Typhoid Fever and Associated Prevailing Drug Resistance in Northern Ethiopia," International Journal of Infectious Diseases, vol. 35, pp. e96-e102, 2015.

[31] S. Kariuki, J. Mwituria, A. Munyalo, G. Revathi, and J. Onsongo, "Typhoid is over-reported in Embu and Nairobi, Kenya.," African Journal of Health Sciences, vol. 11, no. 3-4, pp. 103-110, 2004.

[32] F. G. Youssef, A. S. Daba, S. S. Kabeil, and T. M. Parker, "A comparative study of blood culture and antibody response with the duration of Illness in diagnosis of typhoid fever," Australian Journal of Basic and Applied Sciences, vol. 4, no. 4, pp. 609-614, 2010.

[33] G. Tadesse, "Prevalence of human Salmonellosis in Ethiopia: a systematic review and meta-analysis," BMC Infectious Diseases, vol. 14, article no. 88, 2014.

[34] S. M. Fletcher, D. Stark, and J. Ellis, "Prevalence of gastrointestinal pathogens in sub-saharan africa: Systematic review and meta-analysis," Journal of Public Health in Africa, vol. 2, no. 2, article no. e30, pp. 127-137, 2011.

[35] C. F. Amábile-Cuevas, "Global perspectives of antibiotic resistance," Antimicrobial Resistance in Developing Countries, pp. 313, 2010.

[36] L. Garedew, Z. Hagos, Z. Addis, R. Tesfaye, and B. Zegeye, "Prevalence and antimicrobial susceptibility patterns of Salmonella isolates in association with hygienic status from butcher shops in Gondar town, Ethiopia," Antimicrobial Resistance and Infection Control, vol. 4, no. 1, article 21, 2015.

[37] D. Hailu, A. Gelaw, W. Molla, L. Garedew, L. Cole, and R. Johnson, "Prevalence and Antibiotic Resistance Patterns of Salmonella Isolates from Lactating Cows and In-contact Humans in Dairy Farms, Northwest Ethiopia," Journal of Environmental and Occupational Science, vol. 4, no. 4, p. 171, 2015.

[38] M. Ejo, L. Garedew, Z. Alebachew, and W. Worku, "Prevalence and Antimicrobial Resistance of Salmonella Isolated from Animal-Origin Food Items in Gondar, Ethiopia," BioMed Research International, vol. 2016, Article ID 4290506, 2016.

[39] G. Tadesse, "A meta-analysis of the proportion of antimicrobial resistant human Salmonella isolates in Ethiopia," BMC Pharmacology \& Toxicology, vol. 15, no. 1, article no. 51, 2014. 


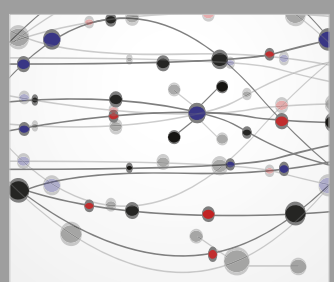

The Scientific World Journal
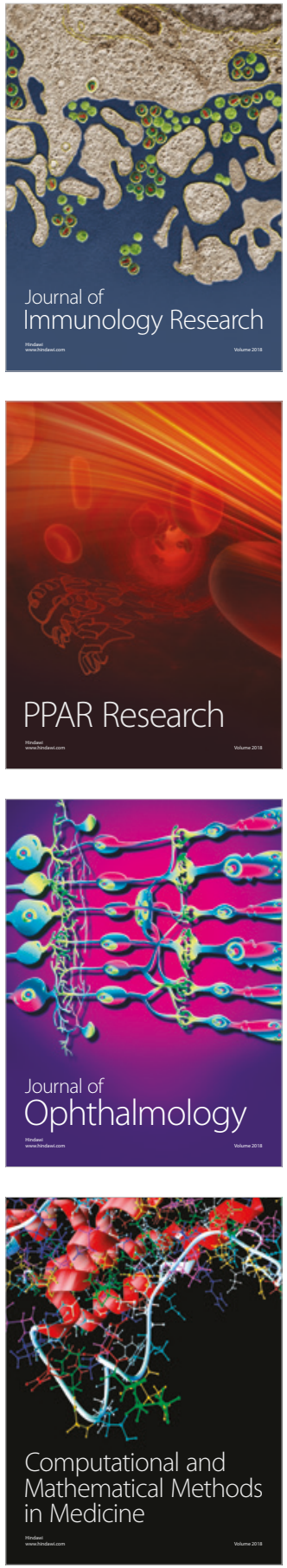

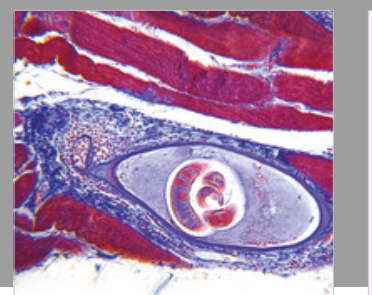

Gastroenterology Research and Practice

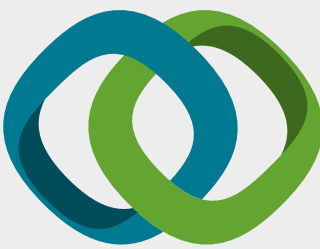

\section{Hindawi}

Submit your manuscripts at

www.hindawi.com
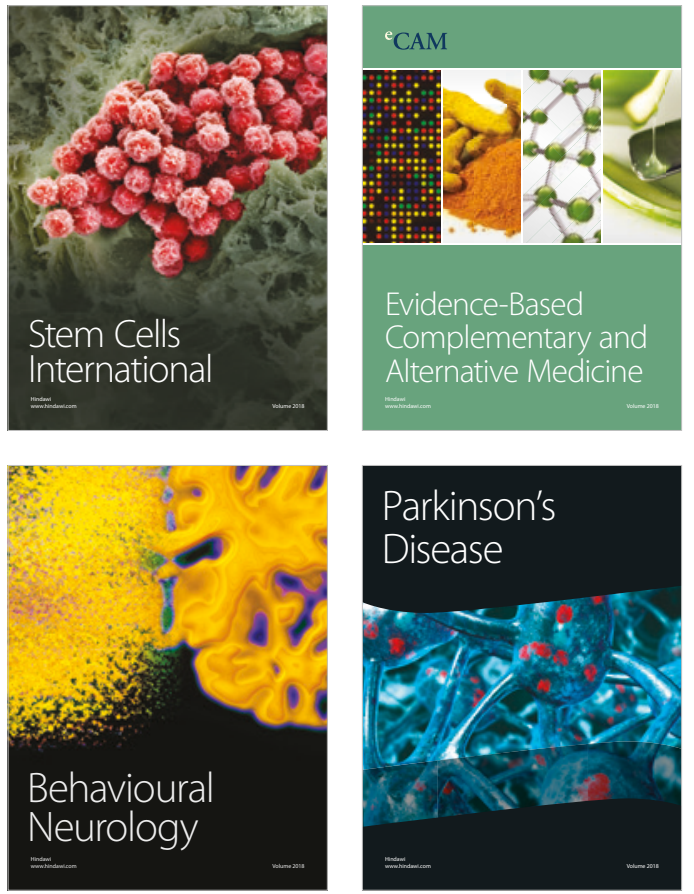

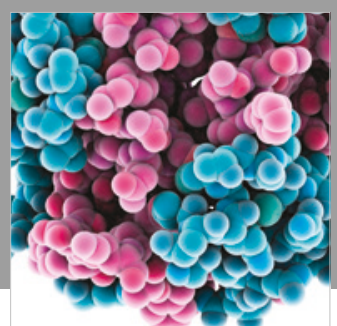

ournal of

Diabetes Research

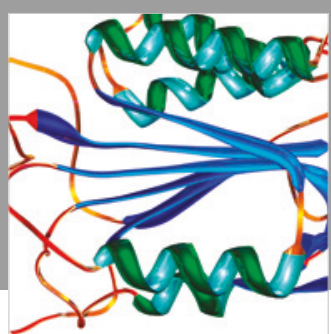

Disease Markers
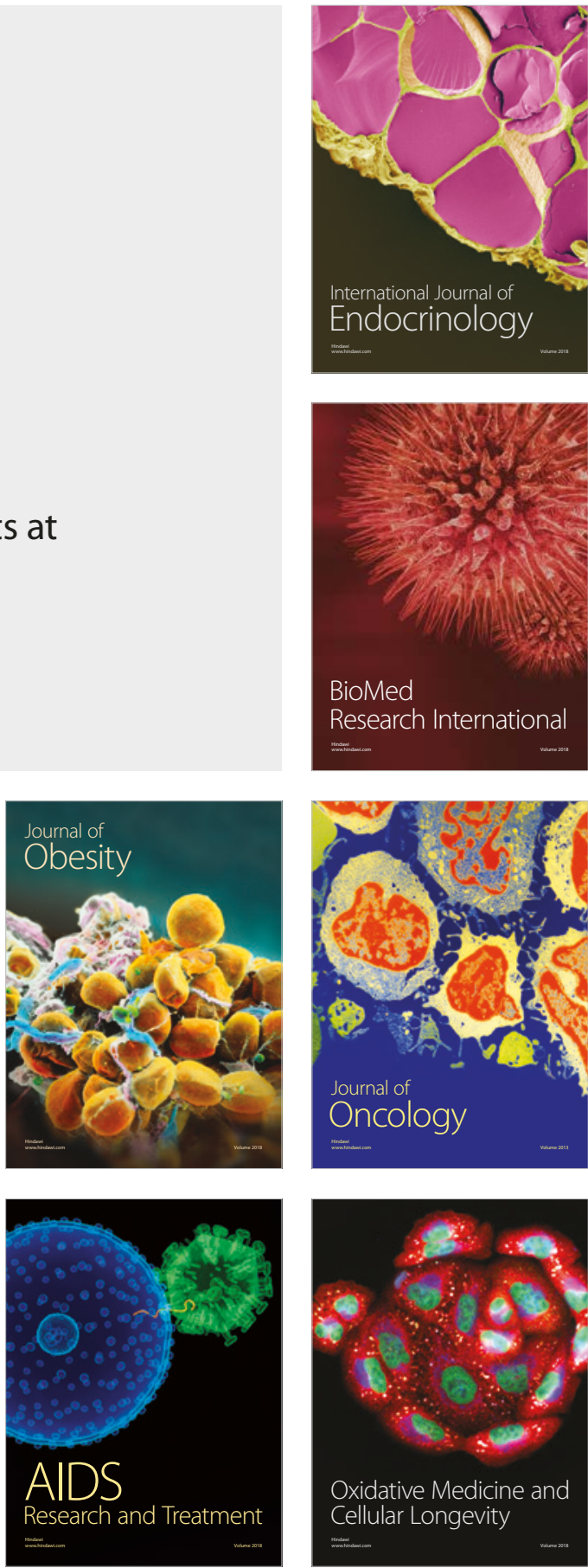